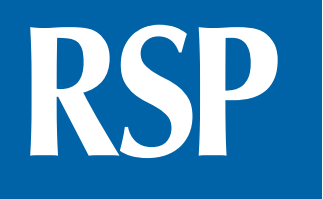

http://www.rsp.fsp.usp.br/
Revista de Saúde Pública

\title{
Fatores associados aos transtornos mentais comuns: estudo baseado em clusters de mulheres
}

\author{
Cássio Zottis Grapiglia' iD, Juvenal Soares Dias da Costa" iD, Marcos Pascoal Pattussi" iD, Vera \\ Maria Vieira Paniz" iD, Maria Teresa Anselmo Olinto" \\ ' Universidade do Vale do Rio dos Sinos. Programa de Saúde Coletiva. São Leopoldo, RS, Brasil \\ " Universidade do Vale do Rio dos Sinos. Departamento de Saúde Coletiva. São Leopoldo, RS, Brasil
}

\section{RESUMO}

OBJETIVO: Identificar fatores associados aos transtornos mentais comuns (TMC) em uma amostra de mulheres adultas no Sul do Brasil.

MÉTODOS: Em estudo de base populacional composto por 1.128 mulheres, foram investigadas variáveis explicativas demográficas, socioeconômicas, comportamentais e de saúde/doença. Cinco grupos-resposta foram explorados: um grupo com transtornos mentais comuns - ponto de corte 6/7 no Self-Reporting Questionnaire 20 (SRQ-20) - e outros quatro correspondentes aos diferentes clusters encontrados através da técnica de clusterização por classe latente, também a partir do SRQ-20. Esses quatro clusters (baixo, médio-depressivo, médio-digestivo e alto) foram denominados com base nas pontuações médias do SRQ-20 em cada grupo e nos padrões de resposta das variáveis e características fatoriais. O cluster "baixo" compreendeu mulheres com menores escores no SRQ-20 e, portanto, com menor probabilidade de apresentarem TMC. Já o cluster "alto", com valores médios elevados no SRQ-20, esteve relacionado a maior morbidade psiquiátrica. Utilizou-se a técnica de regressão de Poisson, com comparação entre os achados dos diferentes grupos.

Cássio Zottis Grapiglia Rua Humaitá 356, Bairro Centro 95705-018 Bento Gonçalves, RS, Brasil

E-mail:czottis@yahoo.com

Recebido: 4 set 2020

Aprovado: $7 \mathrm{dez} 2020$

Como citar: Grapiglia CZ, Dias da Costa JS, Pattussi MP, Paniz VMV, Olinto MTA. Fatores associados aos transtornos mentais comuns: estudo baseado em clusters de mulheres. Rev Saude Publica. 2021;55:77. https://doi.org/10.11606/s15188787.2021055003124

Copyright: Este é um artigo de acesso aberto distribuído sob os termos da Licença de Atribuição Creative Commons, que permite uso irrestrito, distribuição e reprodução em qualquer meio, desde que o autor e a fonte originais sejam creditados.

RESULTADOS: Foram identificadas 10 variáveis como fatores associados aos TMC. Idade, escolaridade, tabagismo, atividade física, percepção de saúde e número de consultas foram as variáveis comuns para as análises com ponto de corte assim como nas baseadas em clusters. Etilismo pesado esteve associado somente quando avaliada a amostra como ponto de corte. Já classe econômica, situação laboral e presença de doenças crônicas estiveram associadas somente quando analisada a amostra por clusters. No cluster "alto", houve associação significativa com classes econômicas mais baixas (D ouE), tabagismo, inatividade física, presença de doenças crônicas e percepção de saúde negativa.

CONCLUSÕES: Diferentes fatores associados foram identificados segundo os grupos-resposta considerados. Novos enfoques que possibilitem identificar subgrupos de indivíduos com características e fatores associados específicos poderiam contribuir para um entendimento mais apurado dos TMC, servindo de base para intervenções em saúde.

DESCRITORES: Mulheres. Transtornos Mentais, epidemiologia. Fatores de Risco. Fatores Socioeconômicos. Análise de Classes Latentes. 


\section{INTRODUÇÃO}

O termo "transtornos mentais comuns" (TMC) refere-se a um conjunto de sintomas depressivos, insônia, fadiga, irritabilidade, esquecimento e dificuldade de concentração, além de queixas somáticas e sentimento de inutilidade ${ }^{1}$. Esses transtornos, mais prevalentes em mulheres, causam sofrimento e incapacidade funcional, além de impactarem em diversos desfechos de saúde, econômicos e sociais ${ }^{2,3}$.

Foram identificadas prevalências de $17,6 \%$ no ano e $29,2 \%$ na vida em estudo de revisão sistemática e metanálise de Steel et al. ${ }^{4}$ que observou efeito consistente do sexo feminino em relação a transtornos de humor e ansiedade. Estudos realizados no Brasil demonstraram prevalência elevada na atenção primária em saúde ${ }^{5}$ e maior razão de chance de TMC em mulheres ${ }^{6}$.

O Self-Reporting Questionnaire-20 (SRQ-20), um dos instrumentos mais recomendados e utilizados para rastrear transtornos mentais comuns, encontra-se validado inclusive no Brasil $^{7-9}$. Diversos estudos de fatores associados aos TMC utilizaram esse instrumento para definir a presença de morbidade psiquiátrica, baseando-se no critério do ponto de corte. No Brasil, os pontos de corte mais utilizados para as mulheres têm sido $6 / 7^{10} \mathrm{e} 7 / 8^{11}$.

Seguindo esse critério, estudos identificaram que indivíduos de sexo feminino ${ }^{2,3,5,12}$, de cor não branca ${ }^{2,13}$, pertencentes a grupos com desvantagem social (classe social mais baixa e menor renda, desempregados e com escolaridade baixa) ${ }^{2,3,12,14}$, tabagistas ${ }^{13,15}$, etilistas pesados $^{16,17}$, inativos fisicamente ${ }^{13}$, com doenças crônicas ${ }^{13,18}$ e com autopercepção de saúde ruim $^{13,19}$ apresentaram maiores prevalências de TMC. Além disso, a maior utilização dos serviços de saúde, com mais consultas/ano, também foi identificada como marcador de $\mathrm{TMC}^{20}$. A relação da idade ${ }^{2,3,12,13,21}$ e do estado conjugal ${ }^{2,3,13,22}$ com os TMC tem demonstrado resultados contraditórios.

Não foram encontrados estudos explorando fatores associados aos TMC além da tradicional análise de estudos observacionais, que constrói o desfecho a partir de pontos de corte e pode não reconhecer subgrupos ou clusters. O presente estudo propõe-se a investigar fatores associados aos TMC em uma amostra de mulheres adultas no Sul do Brasil, utilizando o ponto de corte e também identificando clusters ou subgrupos pela técnica de análise de classe latente (com o instrumento SRQ-20), seguida de análise de regressão de Poisson.

\section{MÉTODOS}

O estudo transversal "Condições de vida e saúde de mulheres adultas: estudo de base populacional no Vale dos Sinos - avaliação após 10 anos" contemplou amostra de 1.128 mulheres no município de São Leopoldo (RS). A pesquisa teve protocolo aprovado pelo Comitê de Ética da Unisinos sob o ${ }^{\circ} 650.443$. Cada participante foi esclarecida a respeito dos objetivos do estudo e, após leitura e assinatura de termo de consentimento livre e esclarecido em duas vias, procedeu-se a coleta de dados.

Estabeleceram-se como critérios de inclusão: ser mulher residente em algum dos setores censitários e domicílios sorteados da zona urbana de São Leopoldo e ter idade entre 20 e 69 anos. As mulheres que, apesar de estarem no domicílio sorteado à época da pesquisa, não residiam nesse domicílio, assim como aquelas que não apresentaram condições físicas/mentais para responder o questionário, ou que estavam grávidas, foram excluídas.

Foi realizada amostragem por conglomerados, com 40 setores censitários sorteados entre os 371 da zona urbana do município de São Leopoldo. O tamanho da amostra do estudo de base foi calculado para identificar uma razão de risco de 2,0, com um nível de confiança de $95 \%$ e poder estatístico de $80 \%$, mantendo razão de expostos: não expostos de 2:1. Consideraram-se não expostos os pertencentes à categoria de maior escolaridade (15 ou mais anos de estudo). Com base nesses pressupostos, optou-se pelo maior tamanho 
de amostra calculado (1.013 mulheres para a variável exame citopatológico atrasado). Foram acrescentados $10 \%$ para eventuais perdas/recusas e 15\% para controlar fatores de confusão na análise dos dados, totalizando aproximadamente 1.281 mulheres. Após consideradas as perdas e recusas, que corresponderam a 11,9\%, o número total final foi de 1.128 mulheres entrevistadas.

As informações foram coletadas por meio de questões de instrumento construído (abarcando dados demográficos, socioeconômicos e comportamentais sobre saúde/doença, utilização de serviços sanitários, medicamentos e gastos em saúde) e de questões pertencentes a instrumentos validados, destacando-se o SRQ-20, aplicado para rastrear TMC. Os questionários foram aplicados diretamente às residentes, nos domicílios sorteados. Conduziu-se o trabalho de campo com rigor, e os entrevistadores foram submetidos a programa de treinamento com padronização de medidas e estudo-piloto. $\mathrm{O}$ banco de dados foi digitado em duplicidade para posterior comparação. Realizou-se o controle de qualidade por meio de instrumento resumido composto de 10 questões, aplicado em $10 \%$ da amostra, por contato telefônico ou visita domiciliar.

Foram consideradas como variáveis explicativas: idade (20-29, 30-39, 40-49, 50-59, 60 anos ou mais), cor da pele (branca e não branca), situação conjugal (como ou sem parceiro), escolaridade ( 4 anos ou menos de estudo, 5 a 9 anos de estudo, 10 a 12 anos de estudo, 13 anos de estudo ou mais), classe econômica (A ou B, C, D ou E), situação laboral (trabalhando, aposentada/afastada/recebendo benefício, dona de casa, desempregada), ser ou não chefe de família, tabagismo (não fumante, ex-fumante ou fumante), etilismo pesado, definido pela ingestão de mais de 30 gramas de álcool/dia (não ou sim), atividade física de lazer, segundo critério do International Physical Activity Questionnaire ${ }^{23}$ (IPAQ) (fisicamente ativa ou inativa), gestações prévias (nenhuma, uma e duas ou mais), presença de doenças crônicas, referindo-se a diabetes ou hipertensão arterial (nenhuma doença, alguma das doenças, ou as duas doenças), percepção de saúde (positiva ou negativa) e número de consultas por ano (1 consulta, 2-5, 6-13, 14 ou mais consultas).

Para o presente estudo, foi construído um banco de dados resumido, composto somente pelas variáveis de exposição e pelo instrumento SRQ-20. Após a construção do banco referido, identificaram-se clusters a partir dos padrões de respostas do instrumento SRQ-20, por meio de análise de classe latente com o software Latent GOLD 5.1. Essa técnica apresenta vantagens em relação às técnicas de clusterização convencionais ${ }^{24}$. Os critérios para definir o número de clusters foram o bayesiano (BIC), a porcentagem de erro de classificação e a análise de resíduos ${ }^{25}$.

Optou-se por quatro clusters devido ao menor valor de BIC, assim como por um perfil satisfatório entre a porcentagem de erros de classificação e o perfil de resíduos. Além dos critérios mencionados, também se considerou o número proporcional de mulheres em cada cluster. A denominação dada aos clusters se refere a pontuação média do SRQ-20, assim como aos padrões de resposta das variáveis do instrumento.

Também de modo exploratório, realizou-se uma análise fatorial do instrumento, utilizando matriz tetracórica e valores mínimos de 0,3 e 0,4 para cargas fatoriais e comunalidades, respectivamente. O software utilizado foi o FACTOR 10.9.02. Essa análise fatorial identificou dois fatores (Tabela 1). O primeiro fator, denominado "depressivo", foi composto pelas variáveis 6 ("Você se sente nervosa, tensa ou preocupada?"), 9 ("Você tem se sentido triste ultimamente?"), 10 (“Você tem chorado mais do que de costume?"), 11 (“Você consegue sentir algum prazer nas atividades diárias?"), 14 (“Você se sente útil na sua vida?"), 15 (“Você tem perdido o interesse pela vida?"), 16 (“Você se sente uma pessoa sem valor?"), 17 ("Você alguma vez pensou em acabar com a sua vida?"), 18 ("Você se sente cansada o tempo todo?”), 20 (“Você se cansa com facilidade?"). Já o segundo fator, denominado “digestivo", foi composto pelas variáveis 7 (“Você tem má digestão?") e 19 ("Você sente alguma coisa desagradável no estômago?”). 
O cluster 1, ou "baixo", apresentou um SRQ médio de 1,94 (IC95\%: 1,79-2,08), enquanto no cluster 4, ou "alto", a média foi de 13,60 (IC95\%: 13,25-13,93). Os clusters intermediários ("médio-depressivo" ou 2, e "médio-digestivo" ou 3) apresentaram médias próximas de SRQ: 6,08 (IC95\%: 5,87-6,29) e 7,63 (7,36-7,89), respectivamente, porém com um perfil mais positivo para as variáveis representativas do fator depressivo no primeiro grupo e de sintomas digestivos no segundo grupo (Tabela 2). Essas variáveis denominadas "fator depressivo" e "fator digestivo" foram construídas a partir da análise fatorial do instrumento e tiveram valor total máximo de 10 pontos e 2 pontos, respectivamente (Tabelas 1 e 2). Após a identificação dos clusters, esse banco foi migrado para os softwares SPSS e Stata 8.0, onde foram realizadas análises descritivas da amostra, além de análises brutas e ajustadas.

Em relação às variáveis de resposta (grupos-resposta), foram contemplados 5 grupos. O primeiro foi o grupo positivo para TMC, considerando o ponto de corte $6 / 7^{10}$. Utilizou-se esse grupo a modo de referência, como uma forma mais clássica de investigar fatores associados em relação ao assunto. Os outros quatro grupos-resposta foram os quatro clusters descritos. Avaliou-se a associação entre as variáveis explicativas e as variáveis de resposta (grupos-resposta) pelas razões de prevalência (RP) e pelos intervalos de confiança (IC95\%). As análises brutas e ajustadas foram realizadas por meio da regressão de Poisson, com controle para efeito de delineamento. Nas análises ajustadas, incluíram-se inicialmente todas as variáveis, retirando sequencialmente das análises aquelas que não apresentaram valor-p mínimo de 0,05 . Por fim, permaneceram somente as variáveis significativas em cada um dos cinco grupos-resposta considerados.

Tabela 1. Análise fatorial utilizando matriz tetracórica.

\begin{tabular}{|c|c|c|c|}
\hline Variáveis & $\begin{array}{c}\text { Fator } 1 \\
\text { (Depressivo) }\end{array}$ & $\begin{array}{c}\text { Fator } 2 \\
\text { (Digestivo) }\end{array}$ & Comunalidade \\
\hline 6) Você (a sra.) se sente nervosa, tensa ou preocupada? & 0,468 & 0,267 & 0,416 \\
\hline 7) Você (a sra.) tem má digestão? & -0.108 & 0,888 & 0,702 \\
\hline 9) Você (a sra.) tem se sentido triste ultimamente? & 0,730 & 0,105 & 0,622 \\
\hline 10) Você (a sra.) tem chorado mais do que de costume? & 0.665 & 0,018 & 0,455 \\
\hline $\begin{array}{l}\text { 11) Você (a sra.) consegue sentir algum prazer nas } \\
\text { atividades diárias? }\end{array}$ & $-0,848$ & 0,157 & 0,610 \\
\hline 14) Você (a sra.) se sente útil na sua vida? & $-0,724$ & 0,083 & 0,470 \\
\hline 15) Você (a sra.) tem perdido o interesse pela vida? & 0,936 & $-0,061$ & 0,822 \\
\hline 16) Você (a sra.) se sente uma pessoa sem valor? & 0,854 & $-0,061$ & 0,680 \\
\hline $\begin{array}{l}\text { 17) Você (a sra.) alguma vez pensou em acabar com } \\
\text { sua vida? }\end{array}$ & 0,741 & $-0,106$ & 0,481 \\
\hline 18) Você (a sra.) se sente cansada o tempo todo? & 0,674 & 0,191 & 0,620 \\
\hline $\begin{array}{l}\text { 19) Você (a sra.) sente alguma coisa desagradável } \\
\text { no estômago? }\end{array}$ & -0.070 & 0,971 & 0,879 \\
\hline 20) Você (a sra.) se cansa com facilidade? & 0,578 & 0,284 & 0,580 \\
\hline
\end{tabular}

Tabela 2. Comparação entre os SRQ médio e os fatores digestivo e depressivo, segundo a amostra total e os diferentes grupos considerados $(\mathrm{n}=1.128)$

\begin{tabular}{|c|c|c|c|c|c|c|}
\hline & $\begin{array}{c}\text { Amostra } \\
\text { Total } \\
(\mathrm{n}=1.128)\end{array}$ & $\begin{array}{c}\text { SRQ+ } \\
(\text { Corte 6/7) } \\
(n=450)\end{array}$ & $\begin{array}{c}\text { Cluster } \\
\text { Baixo } \\
(n=452)\end{array}$ & $\begin{array}{c}\text { Cluster } \\
\text { Médio-Depressivo } \\
(n=240)\end{array}$ & $\begin{array}{c}\text { Cluster } \\
\text { Médio-Digestivo } \\
(n=236)\end{array}$ & $\begin{array}{c}\text { Cluster } \\
\text { Alto } \\
(\mathrm{n}=\mathbf{2 0 0})\end{array}$ \\
\hline SRQ média (IC95\%) & $\begin{array}{c}6,07 \\
(5,81-6,34)\end{array}$ & $\begin{array}{c}10,76 \\
(10,47-11,05)\end{array}$ & $\begin{array}{c}1,94 \\
(1,79-2,08)\end{array}$ & $\begin{array}{c}6,08 \\
(5,87-6,29)\end{array}$ & $\begin{array}{c}7,63 \\
(7,36-7,89)\end{array}$ & $\begin{array}{c}13,60 \\
(13,25-13,93)\end{array}$ \\
\hline Fator digestivo ${ }^{a}$ (IC95\%) & $\begin{array}{c}0,64 \\
(0,60-0,69)\end{array}$ & $\begin{array}{c}1,13 \\
(1,05-1,21)\end{array}$ & $\begin{array}{c}0,22 \\
(0,17-0,27)\end{array}$ & $\begin{array}{c}0,03 \\
(0,01-0,04)\end{array}$ & $\begin{array}{c}1,64 \\
(1,57-1,70)\end{array}$ & $\begin{array}{c}1,18 \\
(1,06-1,30)\end{array}$ \\
\hline Fator depressivo ${ }^{\mathrm{b}}(\mathrm{IC} 95 \%)$ & $\begin{array}{c}2,86 \\
(2,71-3,01)\end{array}$ & $\begin{array}{c}5,28 \\
(5,08-5,49)\end{array}$ & $\begin{array}{c}0,69 \\
(0,62-0,76)\end{array}$ & $\begin{array}{c}3,30 \\
(3,14-3,46)\end{array}$ & $\begin{array}{c}2,82 \\
(2,66-2,98)\end{array}$ & $\begin{array}{c}7,30 \\
(7,10-7,49)\end{array}$ \\
\hline
\end{tabular}

a Fator digestivo: variável com valores entre $0-2$, segundo achados fatoriais na amostra.

${ }^{b}$ Fator depressivo: variável com valores entre $0-10$, segundo achados fatoriais na amostra. 
Tabela 3. Análise descritiva das variáveis socioeconômicas, demográficas, comportamentais e de saúde e doença na amostra de mulheres do município de São Leopoldo, Sul do Brasil ( $n=1.128$ ).

\begin{tabular}{|c|c|c|c|c|c|c|}
\hline Grupos & $\mathbf{n}$ & $\begin{array}{c}\% \text { SRQ+ } \\
\text { (Corte 6/7) }\end{array}$ & $\begin{array}{l}\% \text { Cluster } \\
\text { Baixo }\end{array}$ & $\begin{array}{c}\text { \% Cluster } \\
\text { Médio-Depressivo }\end{array}$ & $\begin{array}{c}\% \text { Cluster } \\
\text { Médio-Digestivo }\end{array}$ & $\begin{array}{c}\% \\
\text { Cluster } \\
\text { Alto }\end{array}$ \\
\hline Variáveis & 1.128 & 39,9 & 40,1 & 21,3 & 20,9 & 17,7 \\
\hline \multicolumn{7}{|l|}{ Idade (anos) } \\
\hline $20-29$ & 216 & 18,2 & 20,1 & 18,3 & 19,9 & 17,0 \\
\hline $30-39$ & 244 & 24,2 & 16,8 & 30,8 & 24,6 & 18,0 \\
\hline $40-49$ & 276 & 23,8 & 24,3 & 24,6 & 22,9 & 26,5 \\
\hline $50-59$ & 228 & 19,3 & 23,0 & 14,2 & 18,2 & 23,5 \\
\hline 60 ou mais & 164 & 14,4 & 15,7 & 12,1 & 14,4 & 15,0 \\
\hline \multicolumn{7}{|l|}{ Cor da pele } \\
\hline Branca & 840 & 72,7 & 76,3 & 72,9 & 74,2 & 72,5 \\
\hline Não branca & 288 & 27,3 & 23,7 & 27,1 & 25,8 & 27,5 \\
\hline \multicolumn{7}{|l|}{ Situação conjugal } \\
\hline Com parceiro & 720 & 62,9 & 62,4 & 67,9 & 65,3 & 60,5 \\
\hline Sem parceiro & 408 & 37,1 & 37,6 & 32,1 & 34,7 & 39,5 \\
\hline \multicolumn{7}{|l|}{ Escolaridade (anos) } \\
\hline 13 ou mais & 114 & 4,8 & 14,6 & 8,0 & 11,0 & 2,1 \\
\hline $10-12$ & 391 & 30,6 & 38,4 & 35,9 & 35,2 & 27,1 \\
\hline $5-9$ & 417 & 40,4 & 34,8 & 40,5 & 32,6 & 46,4 \\
\hline 4 ou menos & 188 & 24,3 & 12,1 & 15,6 & 21,2 & 24,5 \\
\hline \multicolumn{7}{|l|}{ Classe econômica } \\
\hline A ou B & 390 & 25,7 & 40,6 & 35,7 & 35,2 & 20,0 \\
\hline $\mathrm{C}$ & 596 & 58,0 & 49,8 & 53,4 & 54,2 & 59,0 \\
\hline D ou $E$ & 136 & 16,3 & 9,6 & 10,9 & 10,6 & 21,0 \\
\hline \multicolumn{7}{|l|}{ Situação laboral } \\
\hline Trabalhando & 637 & 50,3 & 61,9 & 56,5 & 58,5 & 42,5 \\
\hline Aposent./afast. ${ }^{a}$ & 186 & 16,7 & 17,7 & 13,8 & 14,4 & 19,5 \\
\hline Dona de casa & 182 & 18,7 & 13,5 & 17,6 & 14,0 & 23,0 \\
\hline Desempregada & 121 & 14,3 & 6,9 & 12,1 & 13,1 & 15,0 \\
\hline \multicolumn{7}{|l|}{ Chefe de família } \\
\hline Não & 544 & 44,0 & 50,9 & 50,0 & 47,5 & 41,0 \\
\hline Sim & 584 & 56,0 & 49,1 & 50,0 & 52,5 & 59,0 \\
\hline \multicolumn{7}{|l|}{ Tabagismo } \\
\hline Não & 661 & 54,0 & 62,4 & 61,3 & 58,5 & 47,0 \\
\hline Ex-tabagista & 259 & 21,8 & 23,9 & 21,3 & 26,3 & 19,0 \\
\hline Sim & 208 & 24,2 & 13,7 & 17,5 & 15,3 & 34,0 \\
\hline \multicolumn{7}{|l|}{ Etilismo pesado } \\
\hline Não & 1087 & 95,8 & 97,3 & 95,8 & 98,3 & 96,0 \\
\hline Sim & 34 & 4,2 & 2,7 & 4,2 & 1,7 & 4,0 \\
\hline \multicolumn{7}{|l|}{ Atividade física } \\
\hline Sim & 162 & 8,4 & 20,8 & 12,1 & 12,7 & 4,5 \\
\hline Não & 966 & 91,6 & 79,2 & 87,9 & 87,3 & 95,5 \\
\hline \multicolumn{7}{|l|}{ Gestações } \\
\hline Nenhuma & 172 & 11,3 & 18,8 & 15,4 & 15,3 & 7,0 \\
\hline 1 & 254 & 19,8 & 23,7 & 25,4 & 22,0 & 17,0 \\
\hline 2 ou mais & 702 & 68,9 & 57,5 & 59,2 & 62,7 & 76,0 \\
\hline
\end{tabular}


Tabela 3. Análise descritiva das variáveis socioeconômicas, demográficas, comportamentais e de saúde e doença na amostra de mulheres do município de São Leopoldo, Sul do Brasil ( $n=1.128)$. Continuação.

\begin{tabular}{|c|c|c|c|c|c|c|}
\hline \multicolumn{7}{|c|}{ Doenças crônicas } \\
\hline Nenhuma & 785 & 62,7 & 75,5 & 75,3 & 69,8 & 51,8 \\
\hline Uma & 269 & 28,8 & 20,5 & 18,4 & 24,7 & 38,0 \\
\hline Duas & 66 & 8,5 & 4,0 & 6,3 & 5,5 & 10,2 \\
\hline \multicolumn{7}{|c|}{ Percepção saúde } \\
\hline Positiva & 748 & 45,6 & 83,4 & 66,3 & 62,7 & 32,0 \\
\hline Negativa & 380 & 54,4 & 16,6 & 33,8 & 37,3 & 68,0 \\
\hline \multicolumn{7}{|l|}{ Consultas/ano } \\
\hline Nenhuma & 174 & 16,0 & 14,6 & 18,8 & 10,2 & 19,6 \\
\hline 1 & 179 & 11,4 & 20,6 & 15,0 & 11,9 & 11,1 \\
\hline $2-5$ & 277 & 19,6 & 27,9 & 23,3 & 27,5 & 15,0 \\
\hline $6-13$ & 172 & 17,1 & 14,4 & 14,6 & 15,7 & 17,6 \\
\hline 14 ou mais & 325 & 35,9 & 22,6 & 28,3 & 34,7 & 36,7 \\
\hline
\end{tabular}

aposent./afast.: categoria que compreendeu mulheres aposentadas, afastadas, recebendo benefício ou licença-maternidade. Os valores em negrito são referentes a variáveis significativas $(p<0,05)$ em cada grupo considerado.

\section{RESULTADOS}

Ao considerar os diferentes clusters, 40,1\% das mulheres pertenceram ao cluster denominado “baixo", 21,3\% ao cluster “médio-depressivo", 20,9\% ao "médio-digestivo", e 17,7\% ao cluster "alto". Como mostra a Tabela 3, 39,9\% das mulheres apresentaram critério de TMC positivo, segundo o ponto de corte 6/7 no instrumento SRQ-20 (Tabela 3).

Em relação às variáveis socioeconômicas e demográficas, houve predomínio de idades intermediárias, entre 30 e 49 anos, quando considerado o grupo do ponto de corte. Já em relação aos clusters, as idades mais elevadas tenderam a ser mais prevalentes naquelas mulheres com SRQ-20 mais elevado. A cor da pele branca predominou em todos os grupos, assim como a situação conjugal de ter parceiro. Ao se analisar a escolaridade, o grupo com maior estudo (13 anos ou mais) foi o menos prevalente no grupo com SRQ+ em relação ao ponto de corte. Nos clusters, a escolaridade variou inversamente com a maior pontuação do SRQ-20. As classes econômicas menos privilegiadas (D ou E) foram mais prevalentes no cluster "alto", sendo a classe C a mais prevalente em todos os clusters, assim como quando considerado o ponto de corte. Em relação à situação laboral, a condição de estar trabalhando prevaleceu em todos os grupos, e a distribuição de mulheres com a condição de ser chefe de família foi similar, com ligeiro aumento da prevalência nos grupos do ponto de corte e no cluster "alto" (Tabela 3).

Ao se considerar as variáveis comportamentais, as mulheres não tabagistas e fisicamente inativas predominaram em todos os grupos. O etilismo pesado (> 30g de álcool/dia) foi muito pouco prevalente em todos os grupos (Tabela 3).

A maioria das mulheres relatou não apresentar nenhuma doença crônica (diabetes ou hipertensão arterial). A maior parte também relatou duas ou mais gestações. A percepção de saúde positiva variou inversamente com os clusters em relação à maior pontuação no SRQ-20: a maior frequência (83,4\%) foi encontrada no cluster “baixo", e a menor (32\%) no cluster "alto". O indicador do número de consultas/ano variou conforme o grupo, distribuindo-se de maneira mais irregular (Tabela 3).

Nas análises brutas e ajustadas, no cluster de maior proteção para os TMC, que foi denominado “baixo", encontrou-se relação entre idade, escolaridade, situação laboral, atividade física, percepção de saúde e número de consultas/ano (Tabela 4). A classe econômica, o tabagismo, a atividade física, a presença de doenças crônicas e a percepção de saúde estiveram associados ao cluster "alto" (Tabela 4). Nos clusters intermediários, somente dois fatores 
Tabela 4. Análises brutas e ajustadas dos grupos com SRQ+ segundo o ponto de corte 6/7, cluster "baixo" e cluster "alto".

\begin{tabular}{|c|c|c|c|c|c|c|}
\hline \multirow[t]{2}{*}{ Grupos } & \multicolumn{2}{|c|}{$\begin{array}{c}\text { SRQ + } \\
(\text { Corte 6/7) } \\
\end{array}$} & \multicolumn{2}{|c|}{$\begin{array}{c}\text { Cluster } 1 \\
\text { (Baixo) }\end{array}$} & \multicolumn{2}{|c|}{$\begin{array}{c}\text { Cluster } 4 \\
\text { (Alto) }\end{array}$} \\
\hline & RP Bruta & RP Ajustada & RP Bruta & RP Ajustada & RP Bruta & RP Ajustada \\
\hline \multicolumn{7}{|l|}{ Variáveis } \\
\hline \multicolumn{7}{|l|}{ Idade (anos) } \\
\hline $20-29$ & 1 & 1 & 1 & 1 & & \\
\hline $30-39$ & $\begin{array}{c}1,18 \\
(0,92-1,51)\end{array}$ & $\begin{array}{c}1,20 \\
(0,94-1,53)\end{array}$ & $\begin{array}{c}0,74 \\
(0,57-0,96)\end{array}$ & $\begin{array}{c}0,73 \\
(0,56-0,95)\end{array}$ & & \\
\hline $40-49$ & $\begin{array}{c}1,02 \\
(0,79-1,32)\end{array}$ & $\begin{array}{c}0,90 \\
(0,71-1,16)\end{array}$ & $\begin{array}{c}0,95 \\
(0,76-1,17)\end{array}$ & $\begin{array}{c}0,98 \\
(0,79-1,21)\end{array}$ & & \\
\hline $50-59$ & $\begin{array}{c}1,01 \\
(0,74-1,37)\end{array}$ & $\begin{array}{c}0,79 \\
(0,60-1,02)\end{array}$ & $\begin{array}{c}1,08 \\
(0,85-1,37)\end{array}$ & $\begin{array}{c}1,24 \\
(1,00-1,53)\end{array}$ & & \\
\hline 60 ou mais & $\begin{array}{c}1,04 \\
(0,79-1,39)\end{array}$ & $\begin{array}{c}0,73 \\
(0,54-0,99)\end{array}$ & $\begin{array}{c}1,03 \\
(0,79-1,33)\end{array}$ & $\begin{array}{c}1,23 \\
(0,93-1,61)\end{array}$ & & \\
\hline \multicolumn{7}{|c|}{ Escolaridade (anos) } \\
\hline 13 ou mais & 1 & 1 & 1 & 1 & & \\
\hline $10-12$ & $\begin{array}{c}1,87 \\
(1,23-2,86)\end{array}$ & $\begin{array}{c}1,59 \\
(1,07-2,37)\end{array}$ & $\begin{array}{c}0,77 \\
(0,64-0,92)\end{array}$ & $\begin{array}{c}0,90 \\
(0,75-1,08)\end{array}$ & & \\
\hline $5-9$ & $\begin{array}{c}2,32 \\
(1,48-3,62)\end{array}$ & $\begin{array}{c}1,71 \\
(1,12-2,60)\end{array}$ & $\begin{array}{c}0,65 \\
(0,54-0,78)\end{array}$ & $\begin{array}{c}0,84 \\
(0,70-1,00)\end{array}$ & & \\
\hline 4 ou menos & $\begin{array}{c}3,09 \\
(1,92-4,98)\end{array}$ & $\begin{array}{c}2,20 \\
(1,44-3,35)\end{array}$ & $\begin{array}{c}0,50 \\
(0,35-0,73)\end{array}$ & $\begin{array}{c}0,68 \\
(0,48-0,95)\end{array}$ & & \\
\hline
\end{tabular}

Classe econômica

A ou B

C

D ou $\mathrm{E}$

Situação laboral

\begin{tabular}{|c|c|c|}
\hline Trabalhando & 1 & 1 \\
\hline Aposent./afast. ${ }^{a}$ & $\begin{array}{c}0,98 \\
(0,84-1,15)\end{array}$ & $\begin{array}{c}1,06 \\
(0,91-1,24)\end{array}$ \\
\hline Dona de casa & $\begin{array}{c}0,77 \\
(0,59-1,00)\end{array}$ & $\begin{array}{c}0,88 \\
(0,67-1,15)\end{array}$ \\
\hline Desempregada & $\begin{array}{c}0,58 \\
(0,43-0,80)\end{array}$ & $\begin{array}{c}0,70 \\
(0,53-0,92)\end{array}$ \\
\hline
\end{tabular}

Tabagismo

$\begin{array}{lcccc}\text { Não } & 1 & 1 & 1 & 1 \\ \text { Ex-tabagista } & 1,03 & 0,97 & 1,03 & 0,92 \\ & (0,87-1,22) & (0,82-1,14) & (0,77-1,38) & (0,69-1,22) \\ \text { Sim } & 1,43 & 1,13 & 2,30 & 1,67 \\ & (1,24-1,65) & (0,99-1,31) & (1,79-2,95) & (1,30-2,16)\end{array}$

Etilismo pesado

$\begin{array}{ccc}\text { Não } & 1 & 1 \\ \text { Sim } & 1,42 & 1,41 \\ & (1,05-1,90) & (1,11-1,79)\end{array}$

Atividade física

\begin{tabular}{|c|c|c|c|c|c|c|}
\hline Ativo & 1 & 1 & 1 & 1 & 1 & 1 \\
\hline Inativo & $\begin{array}{c}1,82 \\
(1,33-2,49)\end{array}$ & $\begin{array}{c}1,35 \\
(1,00-1,82)\end{array}$ & $\begin{array}{c}0,64 \\
(0,54-0,76)\end{array}$ & $\begin{array}{c}0,78 \\
(0,67-0,92)\end{array}$ & $\begin{array}{c}3,56 \\
(1,87-6,77)\end{array}$ & $\begin{array}{c}2,19 \\
(1,19-4,02)\end{array}$ \\
\hline \multicolumn{7}{|c|}{ Doenças crônicas } \\
\hline Nenhuma & & & & & 1 & 1 \\
\hline Uma & & & & & $\begin{array}{c}2,15 \\
(1,61-2,87)\end{array}$ & $\begin{array}{c}1,49 \\
(1,12-1,98)\end{array}$ \\
\hline Duas & & & & & $\begin{array}{c}2,33 \\
(1,44-3,78)\end{array}$ & $\begin{array}{c}1,36 \\
(0,84-2,21)\end{array}$ \\
\hline
\end{tabular}


Tabela 4. Análises brutas e ajustadas dos grupos com SRQ+ segundo o ponto de corte 6/7, cluster "baixo" e cluster "alto". Continuação.

\begin{tabular}{|c|c|c|c|c|c|c|}
\hline \multicolumn{7}{|c|}{ Percepção saúde } \\
\hline Positiva & 1 & 1 & 1 & 1 & 1 & 1 \\
\hline Negativa & $\begin{array}{c}2,35 \\
(2,06-2,68)\end{array}$ & $\begin{array}{c}2,09 \\
(1,86-2,34)\end{array}$ & $\begin{array}{c}0,39 \\
(0,32-0,49)\end{array}$ & $\begin{array}{c}0,44 \\
(0,35-0,55)\end{array}$ & $\begin{array}{c}4,18 \\
(3,15-5,55)\end{array}$ & $\begin{array}{c}3,14 \\
(2,31-4,27)\end{array}$ \\
\hline \multicolumn{7}{|l|}{ Consultas/ano } \\
\hline Nenhuma & 1 & 1 & 1 & 1 & & \\
\hline 1 & $\begin{array}{c}0,69 \\
(0,52-0,91)\end{array}$ & $\begin{array}{c}0,74 \\
(0,55-1,01)\end{array}$ & $\begin{array}{c}1,37 \\
(1,08-1,74)\end{array}$ & $\begin{array}{c}1,29 \\
(1,02-1,64)\end{array}$ & & \\
\hline $2-5$ & $\begin{array}{c}0,77 \\
(0,61-0,96)\end{array}$ & $\begin{array}{c}0,87 \\
(0,71-1,07)\end{array}$ & $\begin{array}{c}1,20 \\
(0,96-1,50)\end{array}$ & $\begin{array}{c}1,08 \\
(0,86-1,36)\end{array}$ & & \\
\hline $6-13$ & $\begin{array}{c}1,08 \\
(0,87-1,34)\end{array}$ & $\begin{array}{c}1,21 \\
(0,98-1,50)\end{array}$ & $\begin{array}{c}1,00 \\
(0,75-1,33)\end{array}$ & $\begin{array}{c}0,93 \\
(0,70-1,22)\end{array}$ & & \\
\hline 14 ou mais & $\begin{array}{c}1,20 \\
(0,96-1,50)\end{array}$ & $\begin{array}{c}1,19 \\
(0,95-1,50)\end{array}$ & $\begin{array}{c}0,83 \\
(0,63-1,09)\end{array}$ & $\begin{array}{c}0,83 \\
(0,63-1,09)\end{array}$ & & \\
\hline
\end{tabular}

RP: razão de prevalência.

a Aposent./afast.: categoria que compreendeu mulheres aposentadas, afastadas, recebendo benefício ou licença-maternidade.

foram identificados: a idade no cluster “médio-depressivo", e o número de consultas/ano no cluster "médio-digestivo" (Tabela 5).

Quando considerado o grupo do ponto de corte, foi encontrada relação entre idade, escolaridade, tabagismo, etilismo, atividade física, percepção de saúde e número de consultas/ ano. Nesse grupo, as menores razões de prevalência foram encontradas nas mulheres mais idosas e, na categoria de idade entre 30 e 39 anos, evidenciou-se uma prevalência $20 \%$ maior que a categoria de referência (ter entre 20 e 29 anos). No cluster "baixo", houve tendência consistente de aumento da prevalência de mulheres com o aumento da idade, com razão de prevalência de 1,24 no grupo de mulheres entre 50 e 59 anos (Tabela 4).

A escolaridade apresentou-se como fator associado relevante quando considerado o ponto de corte, com prevalência 2,2 vezes maior no grupo entre 0 e 4 anos de estudo em comparação à categoria de referência de haver estudado 13 anos ou mais. No cluster "baixo", essa variável também foi significativa, ficando constatada a menor razão de prevalência $(0,68)$ entre as mulheres com menor estudo, o que demonstra efeito protetor da variável em relação aos TMC. Já a classe econômica apresentou associação somente no cluster "alto", com razão de prevalência de 1,76 nas classes mais baixas (D ou E). Outra variável socioeconômica relacionada, a situação laboral, evidenciou tendência linear inversa no sentido de trabalho-desemprego no cluster "baixo", com menor razão de prevalência $(0,70)$ e maior significância entre as desempregadas (Tabela 4).

Na variável comportamental que considerou o tabagismo, as razões de prevalência foram significativas na categoria dos tabagistas, tanto no grupo do ponto de corte $(\mathrm{RP}=1,13)$ quanto no cluster "alto" (RP = 1,67). Já o etilismo pesado (consumo > 30 gramas de álcool/dia) somente esteve associado ao grupo do ponto de corte, com uma razão de prevalência de 1,41.

Em relação à atividade física, houve associação entre ser inativo fisicamente nos grupos do ponto de corte $(\mathrm{RP}=1,35)$, cluster "baixo" $(\mathrm{RP}=0,78)$ e cluster "alto" $(\mathrm{RP}=2,19)$, com todos os intervalos de confiança consistentes (Tabela 4). Da mesma forma e com significância, evidenciou-se também associação entre a variável percepção de saúde nos três grupos. A menor razão de prevalência de uma percepção negativa foi encontrada no cluster "baixo" $(\mathrm{RP}=0,44)$, e a maior no cluster "alto" $(\mathrm{RP}=3,14)$, com valor intermediário no grupo do ponto de corte $(\mathrm{RP}=2,09)$ (Tabela 4).

A presença de doenças crônicas apresentou significância somente no cluster "alto", com prevalência ligeiramente maior na categoria "apresentar uma doença crônica" $(\mathrm{RP}=1,49)$ em comparação a "apresentar duas" (RP = 1,36) (Tabela 4). 
Tabela 5. Fatores associados em relação aos clusters intermediários.

\begin{tabular}{|c|c|c|c|c|}
\hline \multirow[t]{2}{*}{ Grupos } & \multicolumn{2}{|c|}{$\begin{array}{c}\text { Cluster } 2 \\
\text { (Médio-Depressivo) } \\
\end{array}$} & \multicolumn{2}{|c|}{$\begin{array}{c}\text { Cluster } 3 \\
\text { (Médio-Digestivo) } \\
\end{array}$} \\
\hline & RP Bruta & RP Ajustada & RP Bruta & RP Ajustada \\
\hline \multicolumn{5}{|l|}{ Variáveis } \\
\hline \multicolumn{5}{|l|}{ Idade (anos) $^{\mathrm{a}}$} \\
\hline $20-29$ & 1 & 1 & & \\
\hline $30-39$ & $\begin{array}{c}1,49 \\
(1,06-2,09)\end{array}$ & $\begin{array}{c}1,49 \\
(1,06-2,09)\end{array}$ & & \\
\hline $40-49$ & $\begin{array}{c}1,05 \\
(0,73-1,51)\end{array}$ & $\begin{array}{c}1,05 \\
(0,73-1,51)\end{array}$ & & \\
\hline $50-59$ & $\begin{array}{c}0,73 \\
(0,48-1,12)\end{array}$ & $\begin{array}{c}0,73 \\
(0,48-1,12)\end{array}$ & & \\
\hline 60 ou mais & $\begin{array}{c}0,87 \\
(0,57-1,33)\end{array}$ & $\begin{array}{c}0,87 \\
(0,57-1,33)\end{array}$ & & \\
\hline \multicolumn{5}{|l|}{ Consultas/ano ${ }^{\mathrm{b}}$} \\
\hline Nenhuma & & & 1 & 1 \\
\hline 1 & & & $\begin{array}{c}1,13 \\
(0,69-1,85)\end{array}$ & $\begin{array}{c}1,13 \\
(0,69-1,85)\end{array}$ \\
\hline $2-5$ & & & $\begin{array}{c}1,70 \\
(1,08-2,67)\end{array}$ & $\begin{array}{c}1,70 \\
(1,08-2,67)\end{array}$ \\
\hline $6-13$ & & & $\begin{array}{c}1,56 \\
(0,96-2,52)\end{array}$ & $\begin{array}{c}1,56 \\
(0,96-2,52)\end{array}$ \\
\hline 14 ou mais & & & $\begin{array}{c}1,83 \\
(1,12-2,98)\end{array}$ & $\begin{array}{c}1,83 \\
(1,12-2,98)\end{array}$ \\
\hline
\end{tabular}

RP: razão de prevalência.

a Única variável significativa no cluster 2.

búnica variável significativa no cluster 3.

No que se refere às consultas médicas, no grupo do ponto de corte constatou-se tendência de prevalências menores, em relação à referência (nenhuma consulta), nas categorias entre 1 e 5 consultas; e prevalências crescentes em relação a um maior número de consultas (6 ou mais consultas). No cluster "baixo", houve significância nas categorias de uma consulta, e de 14 ou mais consultas, com razões de prevalência de 1,29 e 0,83, respectivamente (Tabela 4). No cluster “médio-digestivo", houve tendência de razões de prevalência progressivas no mesmo sentido das categorias com maior número de consultas. $\mathrm{O}$ maior valor encontrado $(\mathrm{RP}=1,83)$ foi aquele correspondente à categoria com mais consultas: 14 ou mais (Tabela 5).

Também foram investigadas as médias do SRQ-20 em cada um dos grupos analisados, assim como dos fatores digestivo (variáveis 7 e 19) e depressivo (variáveis 6, 9, 10, 11, 14, 15, 16, 17, 18 e 20), definidos segundo análise fatorial da amostra (Tabelas 1 e 2). Em relação ao fator digestivo, observou-se que o cluster "médio-digestivo" foi o que apresentou a maior pontuação nesse fator, maior inclusive que o cluster “alto". Já o cluster “médio-depressivo" foi o que apresentou a menor pontuação nesse fator, menor até que o cluster "baixo" (Tabela 2).

\section{DISCUSSÃO}

A literatura aponta associação significativa entre TMC e o sexo feminino. Os transtornos de humor e a ansiedade são mais prevalentes em mulheres em comparação com os homens, que por sua vez têm mais chances de apresentar transtornos por uso de substâncias ${ }^{4}$. Estudos que pretendem identificar fatores associados aos TMC com instrumentos de rastreamento de saúde mental e seus padrões de resposta, partindo de bases populacionais específicas de mulheres, agregam dados a esse contexto. Quando considerada a visão clássica da análise dos fatores associados aos TMC a partir de um ponto de corte, deve-se ter presente que, dentro dos grupos sem presença de doença (SRQ-) ou com presença de 
doença (SRQ+) - no caso do presente estudo representada pela morbidade psiquiátrica -, podem existir diferentes subgrupos ou clusters, com características particulares e fatores associados específicos.

A utilização do tradicional ponto de corte pode fazer que fatores associados sejam atribuídos ou identificados em grupos que, mesmo sendo muito heterogêneos, são "homogeneizados" por essa metodologia. A avaliação por subgrupos ou clusters baseados nos padrões de respostas do SRQ-20 possibilitou vislumbrar em qual grupo cada fator associado apresentou relevância. Essa nova visão, portanto, pode trazer mais especificidade aos estudos sobre TMC e fatores associados, questionando o tradicional ponto de corte.

Ao considerar a idade como fator associado aos TMC, a literatura tem demonstrado resultados contraditórios ${ }^{2,3,12,13,21}$. A maioria dos estudos revisados observou aumento consistente da prevalência de TMC com a idade, o que não foi constatado no presente estudo. Os resultados aqui encontrados provavelmente refletem as demandas crescentes e o aumento do estresse entre mulheres mais jovens ${ }^{13}$. Devido à análise utilizada, que contemplou clusters, constatou-se efeito protetor da idade em relação aos TMC, com tendência de aumento da presença de mulheres com idades mais elevadas no grupo de menor pontuação do SRQ-20 (cluster "baixo"). Já em clusters com maior probabilidade de apresentar TMC, a tendência se mostrou inversa. Assim, houve redução das prevalências de mulheres com idades mais avançadas a medida que as pontuações no SRQ-20 progrediram (cluster "médio-depressivo" e grupo do ponto de corte).

Estudos têm mostrado que desvantagens sociais como baixo nível educacional, renda, classe econômica e desemprego permanecem sendo os fatores de risco mais consistentes para os $\mathrm{TMC}^{2,3,12,14}$. Apesar da classe econômica e da escolaridade serem variáveis relacionadas, neste estudo foi constatada influência diferente dessas duas variáveis quando avaliados os clusters. Assim, a maior escolaridade apresentou efeito protetor para os TMC, evidenciado no cluster "baixo", assim como o contexto de histórico laboral. A literatura descreve que, entre as mulheres, haveria um efeito protetor do ato de trabalhar sobre os TMC, em comparação com a condição de ser dona de casa ou estar desempregada ${ }^{3,21}$. A classe econômica foi a única variável socioeconômica identificada no grupo com maior probabilidade de morbidade psiquiátrica (cluster "alto"), revelando tendência de maiores prevalências entre as classes econômicas mais baixas, com razão de prevalência significativa de 1,76 nas classes D ou E.

Já no grupo do ponto de corte, somente a escolaridade teve importância como variável socioeconômica, com evidência de aumento das prevalências de maneira consistente à medida que a escolaridade diminui. Existiria, portanto, maior sofrimento mental entre mulheres com piores condições econômicas ou desfavorecidas socialmente. Somadas essas informações, pode-se supor que menores taxas de desemprego, aumento da escolaridade e ascensão às classes econômicas mais favorecidas melhorariam a saúde mental. Cada uma dessas variáveis apresenta uma influência diferente em cada grupo considerado.

Em relação ao tabagismo, a análise apresentou significância na categoria das mulheres atualmente tabagistas no grupo do ponto de corte, corroborando outros estudos realizados no Sul do Brasil ${ }^{13,20}$. No cluster "alto", também foi encontrada essa associação, porém com maior magnitude. Assim, infere-se que a influência do tabagismo se deu sobretudo entre mulheres com o SRQ mais elevado, e não nos valores intermediários, como poderia sugerir a metodologia do ponto de corte.

Estudos também identificaram o etilismo e sua relação com os $\mathrm{TMC}^{13,20,26}$. Com o critério do ponto de corte, houve associação significativa entre os TMC e a variável comportamental do etilismo pesado (> 30g de álcool/dia). Na análise segundo os clusters, essa relação não foi evidenciada, demonstrando que a análise baseada no tradicional ponto de corte encontrou resultado diferente devido ao fato de submeter mulheres com diferentes valores de SRQ+ (que poderiam variar desde 7 até 20 ) a uma mesma análise. 
As variáveis de atividade física e de percepção de saúde foram significativas tanto no grupo do ponto de corte quanto nos clusters extremos ("baixo" e "alto"). Conforme descrito na literatura, encontrou-se efeito benéfico da atividade física em relação ao bem-estar físico e psicossocial, o que se reflete nas razões de prevalência no cluster "baixo". O incremento muscular e a redução da porcentagem de gordura, a otimização das condições cardiorrespiratórias, a redução da ansiedade e da depressão - que impactam no humor e na autoestima - seriam alguns dos efeitos protetores contra os TMC e diversas doenças crônicas ${ }^{13}$. Corroborando outros estudos, foi encontrada maior prevalência de mulheres fisicamente inativas nos grupos do ponto de corte e no cluster "alto", que apresentaram maior morbidade psiquiátrica ${ }^{13,26}$. A autopercepção de saúde considerada negativa apresentou maiores razões de prevalência nos grupos com maior pontuação no SRQ-20 (ponto de corte e cluster “alto"), indo ao encontro dos achados da literatura ${ }^{2,13,21}$.

Em relação a presença de doenças crônicas, pesquisa internacional realizada pela Organização Mundial da Saúde (OMS) em 17 países revelou associação entre os TMC e diversas patologias crônicas como diabetes, asma, hipertensão, artrite, úlcera e doenças cardíacas $^{27}$. Apesar da literatura apontar associação entre os TMC e condições crônicas com o tradicional ponto de corte, o presente estudo observou associação com essas condições apenas no grupo com SRQ mais elevado ("alto"). Esse achado sugere que medidas que otimizem o manejo de doenças crônicas podem ter impacto entre mulheres com maior morbidade psiquiátrica. No cluster "alto", ficou claramente caracterizada a concomitância de fatores comportamentais (tabagismo e inatividade física) com variáveis de saúde e doença (autopercepção de saúde negativa e presença de doenças crônicas).

O cluster com maior pontuação no fator digestivo ("médio-digestivo") apresentou relação progressiva com o número de consultas. Segundo Bekhuis et al. ${ }^{28}$, existem diferentes combinações entre sintomas depressivos, ansiosos e somáticos, o que impacta na demanda por serviços de saúde. Nessa amostra, os sintomas digestivos motivaram um maior número de consultas médicas do que a própria pontuação do SRQ-20 ou sintomas depressivos. Assim, deveria haver um olhar especial para esses sintomas digestivos, atentando para interpretações complexas decorrentes dos sintomas expressados, de modo a compreender melhor essa demanda.

Em resumo, considerando os fatores associados encontrados, o cluster "baixo"é formado majoritariamente por mulheres de idade mais elevada, com maior escolaridade e histórico laboral, fisicamente ativas, com autopercepção de saúde positiva e tendência de se consultar poucas vezes com o médico. O cluster "alto" é composto por mulheres pertencentes a classes sociais mais baixas, tabagistas, inativas fisicamente, com presença de doenças crônicas e autopercepção de saúde negativa. Mulheres com tendência de procurar mais por consultas médicas, valores de SRQ-20 intermediário e maior expressão de sintomas digestivos em geral pertencem ao cluster médio-digestivo. Já o cluster médio-depressivo, também com valores intermediários no SRQ-20, é caracterizado por mulheres jovens, com relato de sintomas mais condizentes com esfera depressiva. O grupo do ponto de corte é formado por mulheres mais jovens, com menor escolaridade, etilistas pesadas e tabagistas, inativas fisicamente, com autopercepção de saúde ruim e tendência maior de procura por consultas médicas.

Como limitações do presente estudo, pode-se mencionar a impossibilidade de se estender seus achados à população em geral, haja vista que a amostra esteve restrita a mulheres. Há também possibilidade de causalidade reversa, visto que se trata de estudo de delineamento transversal. Apesar disso, estudos desse tipo são ferramentas importantes para identificar e descrever grupos de risco, contribuindo para o planejamento de ações em saúde ${ }^{29,30}$.

\section{CONCLUSÃO}

Este estudo, que utilizou a análise de cluster por classe latente, apresenta uma perspectiva nova e diferenciada em relação aos TMC e fatores associados. A caracterização de 
subgrupos, definidos segundo o perfil de resposta do instrumento SRQ-20, permitiu encontrar fatores associados particulares, dando mais especificidade aos resultados. Nessa perspectiva e considerando a relevância do assunto, são necessários mais estudos com essa metodologia.

\section{REFERÊNCIAS}

1. Goldberg DP, Huxley P. Common mental disorders: a bio-social model. New York: Tavistock Books; 1992.

2. Nunes MA, Pinheiro AP, Bessel M, Brunoni AR, Kemp AH, Benseñor IM, et al. Common mental disorders and sociodemographic characteristics: baseline findings of the Brazilian Longitudinal Study of Adult Health (ELSA-Brasil). Braz J Psychiatry. 2016;38(2):91-7. https://doi.org/10.1590/1516-4446-2015-1714

3. Silva M, Loureiro A, Cardoso G. Social determinants of mental health: a review of the evidence. Eur J Psychiatr. 2016;30(4):259-92.

4. Steel Z, Marnane C, Iranpour C, Chey T, Jackson JW, Patel V, et al. The global prevalence of common mental disorders: a systematic review and meta-analysis 1980-2013. Int J Epidemiol. 2014;43(2):476-93. https://doi.org/10.1093/ije/dyu038

5. Gonçalves DA, Mari JJ, Bower P, Gask L, Dowrick C, Tófoli LF, et al. Brazilian multicentre study of common mental disorders in primary care: rates and related social and demographic factors. Cad Saude Publica. 2014;30(3):623-32. https://doi.org/10.1590/0102-311x00158412

6. Coutinho LMS, Matijasevich A, Scazufca M, Menezes PR. Prevalence of common mental disorders and the relationship to the social context: multilevel analysis of the São Paulo Ageing \& Health Study (SPAH). Cad Saude Publica. 2014;30(9):1875-83. https://doi.org/10.1590/0102-311X00175313

7. Ali GC, Ryan G, De Silva MJ. Validated screening tools for common mental disorders in low and middle income countries: a systematic review. PLoS One. 2016;11(6):e0156939. https://doi.org/10.1371/journal.pone.0156939

8. Iacoponi E, Mari JJ. Reliability and factor structure of the Portuguese version of Self-Reporting Questionnaire. Int J Soc Psychiatry. 1989;35(3):213-22. https://doi.org/10.1177/002076408903500301

9. Westhuizen C, Wyatt G, Williams JK, Stein DJ, Sorsdahl K. Validation of the Self Reporting Questionnaire 20-Item (SRQ-20) for use in a low- and middle-income country emergency centre setting. Int J Ment Health Addict. 2016;14(1):37-48. https://doi.org/10.1007/s11469-015-9566-x

10. Santos KOB, Araújo TM, Pinho PS, Silva ACC. Avaliação de um instrumento de mensuração de morbidade psíquica: estudo de validação do Self-Reporting Questionnaire (SRQ-20). Rev Baiana Saude Publica. 2010;34(3):544-60. https://doi.org/10.22278/2318-2660.2010.v34.n3.a54

11. Gonçalves DM, Stein AT, Kapczinski F. Avaliação de desempenho do Self-Reporting Questionnaire como instrumento de rastreamento psiquiátrico: um estudo comparativo com o Structured Clinical Interview for DSM-IV-TR. Cad Saude Publica. 2008;24(2):380-90. https://doi.org/10.1590/S0102-311X2008000200017

12. Patel V, Araya R, Lima M, Ludermir A, Todd C. Women, poverty and common mental disorders in four restructuring societies. Soc Sci Med. 1999;49(11):1461-71. https://doi.org/10.1016/s0277-9536(99)00208-7

13. Moraes RSM, Silva DAS, Oliveira WF, Peres MA. Social inequalities in the prevalence of common mental disorders in adults: a population-based study in Southern Brazil. Rev Bras Epidemiol. 2017;20(1):43-56. https://doi.org/10.1590/1980-5497201700010004

14. Patel V, Kleinman A. Poverty and common mental disorders in developing countries. Bull World Health Organ. 2003;81(8):609-15.

15. Soares PSM, Meucci RD. Epidemiologia dos transtornos mentais comuns entre mulheres na zona rural de Rio Grande, RS, Brasil. Cienc Saude Coletiva. 2020;25(8):3087-95. https://doi.org/10.1590/1413-81232020258.31582018

16. Silva Júnior FJG, Monteiro CFS. Uso de álcool, outras drogas e sofrimento mental no universo feminino. Rev Bras Enferm. 2020;73(1):1-8. https://doi.org/10.1590/0034-7167-2018-0268 
17. Soldera M, Ribeiro CL. Consumo e dependência de álcool. In: Barros MBA, Cesar CLG, Carandina L, Goldbaum M, organizadores. As dimensões da saúde: inquérito populacional em Campinas. São Paulo: Hucitec; 2008.

18. Andrade LH, Benseñor IM, Viana MC, Andreoni S, Wang YP. Clustering of psychiatric and somatic illnesses in the general population: multimorbidity and socioeconomic correlates. Braz J Med Biol Res. 2010;43(5):483-91. https://doi.org/10.1590/s0100-879x2010007500024

19. Soni A, Fahey N, Byatt N, Prabhakaran A, Simas TAM, Vankar J, et al. Association of common mental disorder symptoms with health and healthcare factors among women in rural western India: results of a cross-sectional survey. BMJ Open. 2016;6(7):e010834. https://doi.org/10.1136/bmjopen-2015-010834

20. Costa JSD, Menezes AMB, Olinto MTA, Gigante DP, Macedo S, Brito MAP, et al. Prevalência de distúrbios psiquiátricos menores na cidade de Pelotas, RS. Rev Bras Epidemiol. 2002;5(2):163. https://doi.org/10.1590/S1415-790X2002000200004

21. Senicato C, Azevedo RCS, Barros MBA. Common mental disorders in adult women: identifying the most vulnerable segments. Cienc Saude Coletiva. 2018;23(8):2543-54. https://doi.org/10.1590/1413-81232018238.13652016

22. Lindström M, Rosvall M. Marital status, social capital, economic stress, and mental health: a population-based study. J Soc Sci. 2012;49(3):339-42. https://doi.org/10.1016/j.soscij.2012.03.004

23. Matsudo S, Araújo T, Matsudo V, Andrade D, Andrade E, Oliveira LC, et al. Questionário Internacional de Atividade Física (IPAQ): estudo de validade e reprodutibilidade no Brasil. Rev Bras Ativ Fis Saude. 2001;6(2):5-18.

24. Magidson J, Vermunt JK. Latent class models for clustering: a comparison with K-means. Can J Mark Res. 2002;20(1):36-43.

25. Vermunt JK, Magidson J. Latent class cluster analysis. In: Hagenaars JA, McCutcheon AL, editors. Applied latent class analysis. Cambridge (UK): Cambridge University Press; 2002, p. 89-106.

26. Santos EG, Siqueira MM. Prevalência dos transtornos mentais na população adulta brasileira: uma revisão sistemática de 1997 a 2009. J Bras Psiquiatr. 2010;59(3):238-46 https://doi.org/10.1590/S0047-20852010000300011

27. Scott KM, Bruffaerts R, Tsang A, Ormel J, Alonso J, Angermeyer MC, et al. Depression-anxiety relationships with chronic physical conditions: results from the World Mental Health Surveys. J Affect Disord. 2007;103(1-3):113-20. https://doi.org/10.1016/j.jad.2007.01.015

28. Bekhuis E, Boschloo L, Rosmalen JG, Schoevers RA. Differential associations of specific depressive and anxiety disorders with somatic symptoms. J Psychosom Res. 2015;78(2):116-22. https://doi.org/10.1016/j.jpsychores.2014.11.007

29. Bastos JLD, Durquia RP. Um dos delineamentos mais empregados em epidemiologia: estudo transversal. Sci Med. 2007;17(4):229-32.

30. Zangirolami-Raimundo J, Echeimberg JO, Leone C. Tópicos de metodologia de pesquisa: estudos de corte transversal. J Hum Growth Dev. 2018;28(3):356-60. https://doi.org/10.7322/jhgd.152198

Financiamento: O estudo de base "Condições de Vida e Saúde de Mulheres Adultas: Estudo de Base Populacional no Vale dos Sinos - Avaliação após 10 anos” foi financiado com auxílio do Edital MCTI/CNPQ/Universal 14/2014.

Contribuição dos Autores: Concepção e planejamento do estudo: CZG, JSDC, MTAO. Coleta, análise e interpretação dos dados: CZG, MPP. Elaboração ou revisão do manuscrito: CZG, JSDC, VMVP. Aprovação da versão final: Todos os autores. Responsabilidade pública pelo conteúdo do artigo: Todos os autores.

Conflito de Interesses: Os autores declaram não haver conflito de interesses. 